Revue des patrimoines

\title{
Un dossier de protection monument historique : la demeure dite « abbaye de Roseland » à Nice
}

Jean Marx

\section{(2) OpenEdition}

\section{Journals}

Édition électronique

URL : http://journals.openedition.org/insitu/1195

DOI : 10.4000/insitu. 1795

ISSN : 1630-7305

\section{Éditeur}

Ministère de la culture

Référence électronique

Jean Marx, «Un dossier de protection monument historique : la demeure dite « abbaye de Roseland » à Nice », In Situ [En ligne], 2 | 2002, mis en ligne le 23 avril 2012, consulté le 19 avril 2019. URL : http:// journals.openedition.org/insitu/1195; DOI : 10.4000/insitu.1195

Ce document a été généré automatiquement le 19 avril 2019

\section{(c) (i) () $\Theta$}

In Situ Revues des patrimoines est mis à disposition selon les termes de la licence Creative Commons Attribution - Pas d'Utilisation Commerciale - Pas de Modification 4.0 International. 


\title{
Un dossier de protection monument historique : la demeure dite " abbaye de Roseland » à Nice
}

\author{
Jean Marx
}

1 La première caractéristique de l'étude initiant une procédure de protection au titre des monuments historiques est d'être bien souvent plus subie que voulue. Résultant en l'occurrence d'une demande extérieure, elle ne s'inscrit pas a priori dans le cadre d'une problématique de recherche prédéterminée et pertinente, telles les études patrimoniales conduites par d'autres services ou à l'Université. Ce caractère aléatoire ne doit cependant pas conduire à dévaloriser ce type d'approche. Fréquemment, notamment dans le domaine privé, il permet l'ouverture de portes et autorise l'examen d'édifices demeurés inaccessibles et méconnus.

Pour rendre compte de la diversité et de la complexité du recensement des monuments historiques, l'exemple choisi est celui d'un édifice hétérogène, remanié, multiple presque, associant des aspects étrangers entre eux. Le dossier présenté a été constitué au début 19941. Il porte sur une demeure niçoise nommée "abbaye de Roseland", qui n'a absolument rien à voir avec l'architecture religieuse. Le choix d'un immeuble privé à l'origine ${ }^{2}$ se justifie non seulement par l'importance de cette catégorie dans le corpus des édifices protégés (environ la moitié) et dans les nouvelles demandes, mais aussi par les difficultés spécifiques à l'étude historique de ce patrimoine, moins bien documenté par les sources.

3 Il n'est sans doute pas inutile de rappeler en avant-propos les finalités du dossier de protection monument historique. Contrairement à d'autres, il n'a pas pour objet premier l'enrichissement de la connaissance. Il vise en revanche un double objectif : d'abord servir de support à une procédure de protection juridique, ensuite fournir un état des lieux à un moment donné de la vie d'un édifice, de manière à asseoir son évolution future, les travaux en particulier, sur une documentation la plus complète possible. In fine, le dossier pourra bien sûr revêtir d'autres dimensions et avoir d'autres usages : contribuer à 
l'amélioration de la connaissance, susciter des recherches complémentaires, concourir à la sensibilisation en faveur de l'édifice ... Mais il s'agit d'effets induits, non du but poursuivi.

Reflétant l'intervention faite dans le cadre du séminaire, le propos ne vise pas à une présentation complète et cohérente de l'édifice, telle celle assurée devant les commissions régionale et nationale ${ }^{3}$. L'évocation du monument lui-même sera rapide et partielle. Le sujet n'est pas de décrire complètement l'abbaye de Roseland et de fournir des conclusions définitives. Il n'est pas non plus de promouvoir un modèle de monographie, qui, malgré des tentatives récentes d'homogénéisation ${ }^{4}$, n'existe pas pour diverses raisons au sein du service des monuments historiques.

5 Il s'agira plutôt de proposer le partage d'une expérience pratique, en insistant sur certains problèmes liés à la méthode adoptée, aux sources prospectées, plus ou moins lacunaires, aux résultats inégaux obtenus dans la connaissance de l'édifice en fonction des objectifs poursuivis.

\section{Première approche de l'édifice}

Figure 1

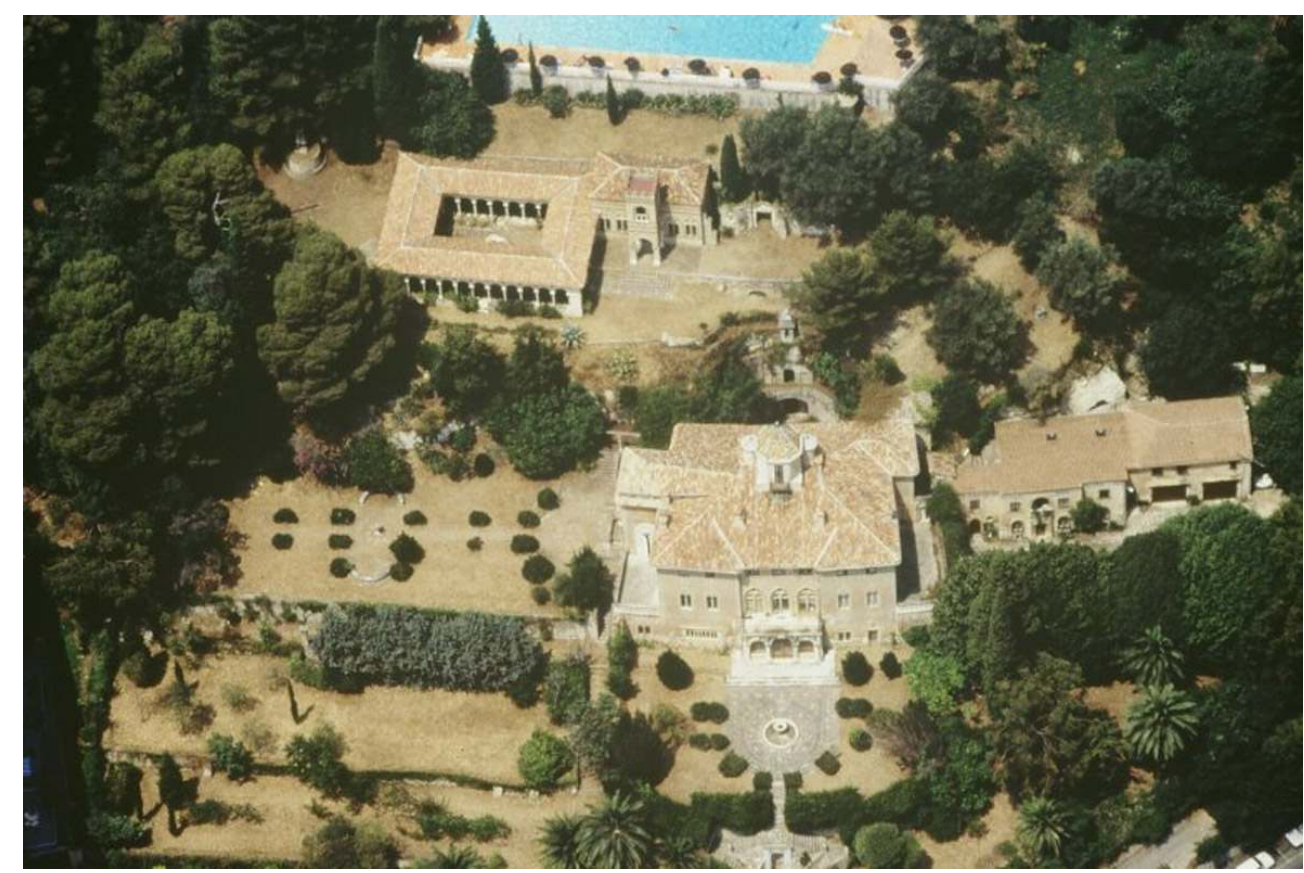

Vue aérienne vers le nord-ouest

Phot. J. Marx (C) Conservation régionale des Monuments historiques PACA., juin 1994

6 Les prémices de la procédure de protection de l'abbaye de Roseland remontent à l'année 1990. A cette date, le service fut saisi d'une demande émanant de l'association de sauvegarde de l'abbaye de Bonnefont-en-Comminges, motivée par la recherche d'éléments lapidaires émigrés de Midi-Pyrénées vers Nice. Une première visite de repérage, discrète, permit d'appréhender le contexte urbain et la situation de propriété : la demeure considérée était enclavée dans un ensemble de barres construites dans les années 60 et elle paraissait bien remployer une importante collection de vestiges sculptés. 
L'année suivante, en 1991, deux demandes de protection furent déposées séparément par la ville et par l'association des copropriétaires de la résidence entourant Roseland.

La première mit en évidence l'intérêt porté par la ville de Nice à ce lieu récemment entré dans son patrimoine. Mais cet intérêt n'était pas totalement dénué d'ambiguïté ; en effet s'était développé depuis 1981 entre le musée archéologique de Cimiez à Nice et le musée des Augustins à Toulouse un projet d'échange de certains chapiteaux de Roseland contre des bracelets du Bronze final provenant de l'oppidum de Cimiez; migrant en sens inverse, les bijoux préhistoriques avaient abouti dans le Midi toulousain. Le processus de restitution réciproque était toutefois au point mort et la ville de Nice avait demandé en 1988 au cabinet d'architecte Février une étude de réhabilitation du domaine de Roseland. Le document produit s'accompagnait de relevés géométraux et consistait surtout en propositions visant à masquer les immeubles voisins par des écrans végétaux.

Le travail de recensement fut réalisé pendant l'été et l'automne 1993. Un premier constat était que les conditions pour une conservation valorisante de Roseland étaient loin d'être réunies. Bien que le domaine fût affecté au service culturel de la ville, l'utilisation comme dépôt de matériels divers était plutôt dégradante pour les bâtiments et leur décor. L'édifice n'était pas en bon état, des travaux de couverture s'avéraient urgents sur la demeure, les collections lapidaires n'étaient nullement à l'abri des risques de dégradation ou de vol, et le tout était plus ou moins à l'abandon. L'enjeu de la protection était donc important: favoriser par l'affirmation de l'intérêt patrimonial de l'édifice l'émergence d'un projet de réutilisation conservatoire et valorisant.

9 L'abbaye de Roseland est située dans la partie ouest de Nice comprise entre le Magnan et le Var. Ce secteur traditionnellement voué aux grands domaines ruraux ne s'est urbanisé que tardivement, en raison d'un relief peu favorable fait de vallons parallèles aux pentes abruptes. A quatre $\mathrm{km}$ de la vieille ville et à $750 \mathrm{~m}$ de la mer, Roseland s'accroche à la pente de la colline de Fabron. Pour une longueur de $90 \mathrm{~m}$ correspondant à la seule emprise du domaine actuel, le dénivelé est de plus de $25 \mathrm{~m}$. Un système de terrasses permet de le compenser. Face à la baie des Anges, la situation de l'édifice demeure agréable; elle était exceptionnelle avant la construction des grands immeubles qui sont venus altérer le paysage. L'environnement végétal reste cependant important car le fonds historique était beaucoup plus étendu que le domaine actuel. Des zones boisées et de grands sujets, en particulier des alignements de cyprès, subsistent du parc ancien.

10 Le domaine originel a donc été morcelé, bâti et ses limites sont aujourd'hui floues. Le dossier ne l'étudie pas dans sa globalité, mais s'en tient à l'actuelle parcelle cadastrale ND 102 qui en était le cœur et qui constitue la propriété de la commune. Il s'agit là d'une première spécificité du dossier de recensement, réductrice sans doute mais imputable à sa finalité. A la différence d'une étude d'inventaire, il doit souvent épouser la situation actuelle du foncier et de la propriété. Dans le cas de Roseland, des vestiges du grand domaine ont certes été repérés, comme l'avenue bordée de cyprès taillés constituant l'entrée de la résidence ou à l'ouest une longue allée forestière aboutissant à un hémicycle de bancs, mais ils ne sont pas pris en compte. 


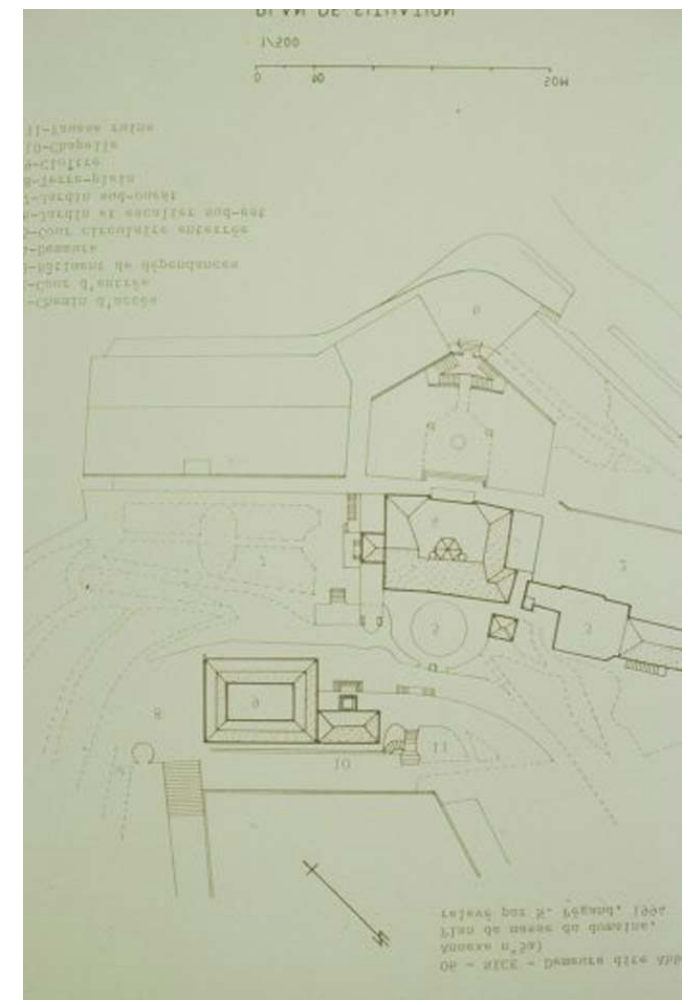

Plan-masse du domaine, relevé par N. Pégand, Inventaire général, 1994

Phot. J. Marx (c) C.R.M.H. PACA., janvier 1996

La parcelle ND 102 est de forme irrégulière à peu près ovoïde ; elle a une superficie de 82 ares et mesure $130 \mathrm{~m}$ par $90 \mathrm{~m}$ dans ses plus grandes dimensions. On accède à la propriété par un chemin partant de l'avenue desservant la résidence et montant à l'est en lacet jusqu'à une cour d'entrée, bordée par les bâtiments de dépendances au nord, par la demeure à l'ouest. Cette dernière occupe une position parfaitement centrale et sa façade antérieure regarde la mer. A l'arrière, une cour circulaire enterrée, coincée entre la façade postérieure et la falaise, commande l'accès à une grotte creusée dans cette dernière. D'autres constructions occupent une terrasse supérieure accessible par un sentier sinueux à l'ouest. Ajoutées au 20e siècle, elles comprennent en particulier un cloitre et une chapelle qui ont justifié le nom d'abbaye de Roseland. Le plan-masse rend compte de cette organisation spatiale et montre les différentes parties constituantes.

Plusieurs axes de composition orthogonaux structurent le domaine et définissent les perspectives majeures. Deux sont parallèles à l'organisation des terrasses : l'un passe par la cour d'entrée, la demeure et le jardin sud-ouest ; l'autre conduit du terre-plein ouest de la terrasse supérieure à une fausse ruine, en passant par le cloître et la chapelle. Deux sont perpendiculaires : le principal, partant du portail d'entrée inférieur, gravit l'escalier du jardin sud-est, traverse le vestibule de la demeure, passe par la cour circulaire arrière et aboutit à la grotte; un autre, décalé vers l'ouest, monte par une allée du jardin, emprunte un escalier, frôle le porche de la façade latérale de la demeure et aboutit au clocher-porche de la chapelle. Des axes secondaires animent les jardins. A cette trame se superpose une organisation concentrique autour de la demeure: les abords immédiats 
sont minéraux (cours, pavements caladés, vestiges lapidaires ...), les espaces plus éloignés accordent une place croissante au végétal.

\section{Les sources de l'étude historique}

13 La grande diversité des dossiers des monuments historiques n'autorise guère l'application d'une méthode systématique. Requiert-elle même une approche particulière? Comme pour d'autres, l'étude implique d'abord une recherche dans les fichiers des bibliothèques spécialisées et dans les dépôts d'archives. Dans le cas de Roseland cette recherche a utilisé des moyens traditionnels, sans recours aux outils informatisés désormais disponibles.

En revanche la relation humaine directe, le réseau relationnel se sont avérés précieux, qu'il s'agisse de partenaires institutionnels, des services de la ville de Nice, du milieu associatif, de chercheurs locaux, de spécialistes nationaux. Les tentatives se soldent parfois malheureusement par un échec, ainsi avec un érudit local qui avait anciennement repéré certaines sources d'archives : celles-ci n'ont pu être retrouvées.

s'avère vite paradoxale : d'un côté il ne faut négliger aucune piste, vérifier toutes les données, croiser les informations recueillies, dans une sorte d'enquête policière qui ne peut avancer que progressivement, où prudence et ténacité sont indispensables. De l'autre, le chercheur a l'obligation d'aller vite, d'être synthétique, de ne pas exploiter les "belles sources". Pour Roseland, les délais impartis ont conduit à l'abandon en cours de route de certaines pistes intéressantes et d'une visite du site avec M. Cabanot, spécialiste de la sculpture médiévale du Sud-Ouest. Achevé, le dossier n'est pas clos; d'autres poursuivront et enrichiront l'étude.

\section{Bibliographie}

Pour l'abbaye de Roseland, un premier repérage bibliographique fournit un état des connaissances initiales, qui s'avérait à la fois riche de promesses et peu fiable, contradictoire. Un article de l'Illustration, de 1929, la présentait comme « une vieille abbaye, ancienne demeure ecclésiastique où vécurent les évêques de Vintimille... une construction du $15 e$ siècle... assez bien conservée pour n'avoir pas besoin de remaniements importants... " ${ }^{5}$. En 1957, un autre journal évoquait "un cloitre datant du 12e siècle découvert aux environs de SaintBertrand-de-Comminges, démonté pierre à pierre " et transporté à Roseland ${ }^{6}$. Plus tard une revue locale précisait qu'elle contenait un "cloitre et une chapelle construits pour réemployer colonnes et chapiteaux du 15e siècle provenant des abbayes de Muret et de Bonnefont-enComminges $»^{7}$.

Les recherches bibliographiques complémentaires mirent en évidence une grande inégalité d'information entre les différentes parties de l'édifice. Sur la demeure ellemême, à peu près rien n'était publié et les quelques mentions éparses n'étaient guère fiables. 
Figure 3

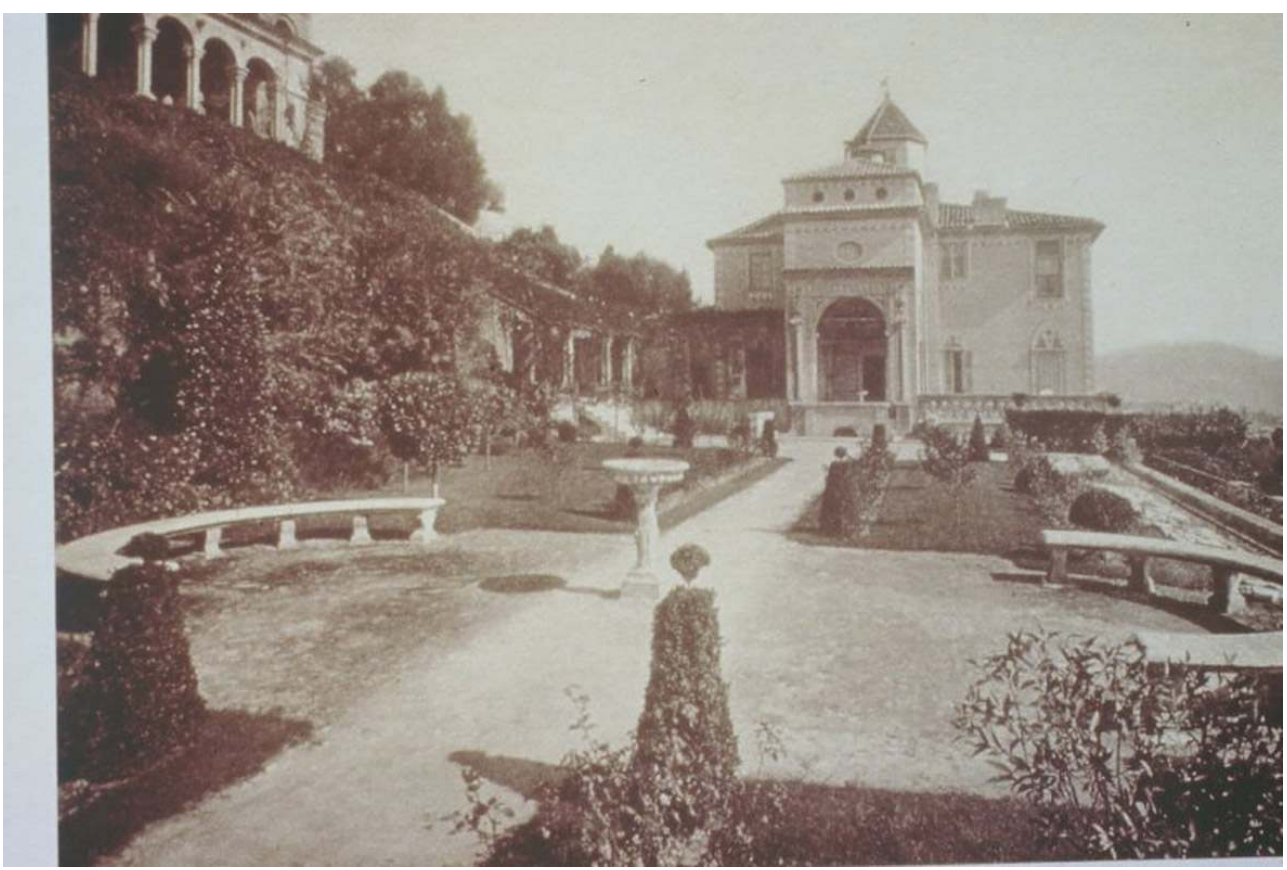

Vue ancienne montrant le jardin ouest, publiée dans O. Godard, Les jardins de la Côte d'Azur, Paris, 1927, pl. I

Repro J. Marx (C) C.R.M.H. PACA., mars 2000

Pour les jardins, peu d'éléments également mais ils étaient précieux; en particulier un ouvrage d'Octave Godard paru en 1927 nous apprenait que ce paysagiste était largement intervenu à Roseland ${ }^{8}$; l'article de l'Illustration déjà cité fournissait quelques photos complémentaires ${ }^{9}$; les jardins avaient enfin fait l'objet d'une analyse sommaire dans le cadre d'un inventaire départemental ${ }^{10}$. 


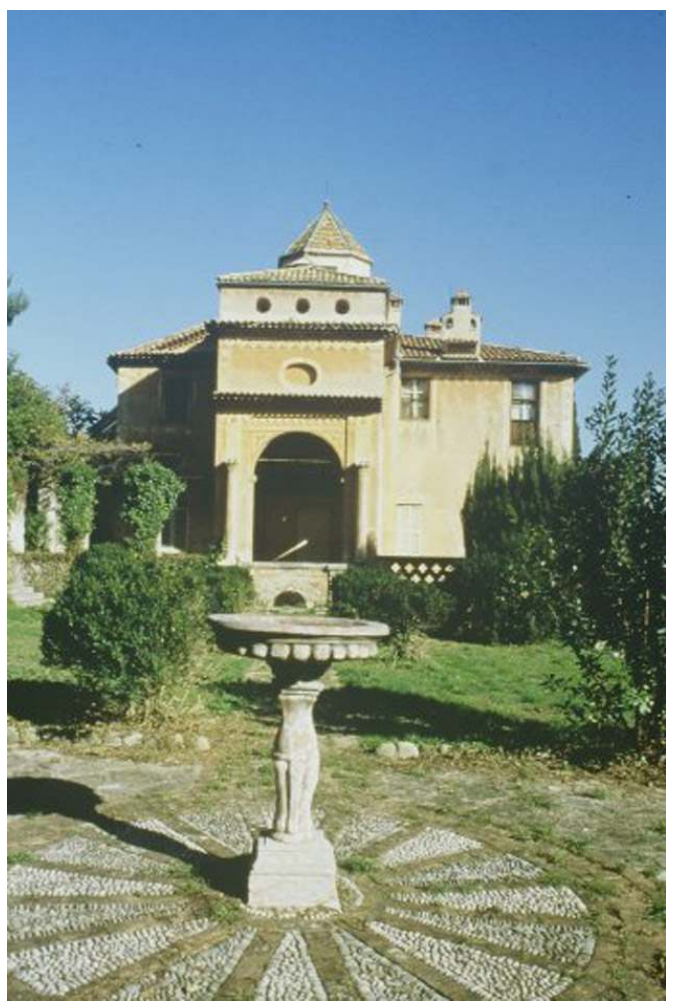

Etat actuel du jardin ouest

Phot. J. Marx (c) C.R.M.H. PACA., novembre 1993

En revanche pour les constructions de la partie haute, réalisées de toutes pièces dans les années 20 par l'antiquaire Larcade à partir d'éléments lapidaires provenant du Sud-Ouest, la bibliographie s'est avérée abondante. Elle concerne parfois directement l'abbaye de Roseland elle-même, mais surtout la sculpture du Midi toulousain. Il n'est pas possible de la citer ici de façon exhaustive, mais seulement de mentionner les principaux textes utilisés dans le dossier sur le problème de la provenance des remplois sculptés. Deux pistes se sont avérées particulièrement fécondes: l'une concerne les sculptures médiévales de la galerie extérieure du pseudo-cloître et de la chapelle ${ }^{11}$, l'autre aborde la question des colonnes et des chapiteaux paléochrétiens de la galerie intérieure du cloître ${ }^{12}$. Si elle ne résout pas la totalité des problèmes liés à la provenance de tous les éléments lapidaires remployés à Roseland, cette bibliographie copieuse permet en tout cas d'avoir aujourd'hui une idée assez précise de la question. Les conclusions en seront données plus loin.

\section{Sources d'archives}

Comme souvent, elles se sont avérées fort laconiques. Cette pauvreté est assez normale pour un édifice privé, domaine par excellence des sources notariales aléatoires. Même pour la période Larcade, aucun document n'a pu être retrouvé éclairant l'histoire des constructions et des remplois. Cette famille a toutefois des descendants et la recherche reste ouverte. 
21 A défaut de fonds privés, les dépôts publics ont été prospectés. Aux Archives départementales des Alpes-Maritimes, le fonds du Sénat de Nice en série B contient des registres regroupant des protocoles ou recueils des minutes des testaments. On y trouve en 1763 un testament par lequel Jean-Ange Dalmassi de Farone lègue sa maison à son cousin le comte Alexandre-Auguste Lascaris ${ }^{13}$. Ce dernier appartenait à une des plus grandes familles niçoises, et son oncle Alexandre Lascaris-Vintimille était prélat. Là se trouve peut-être une des origines de la confusion entretenue par certains auteurs sur la fonction ancienne de Roseland. Hélas certaines sources mentionnées par des chercheurs niçois n'ont pu être vérifiées: les papiers manuscrits de M. Fighera évoquent ainsi un inventaire après décès de 1789 concernant Roseland (maison civile, chambres tapissées d'indienne, maison rurale pour le fermier).

Pour la période révolutionnaire, la série $Q$ n'apporte que de maigres indications, et en raison de l'étendue des propriétés des Lascaris, le doute demeure parfois quant à la localisation exacte des biens évoqués. Il semble cependant qu'une vente de 1796 puisse être rapportée à Roseland. Le domaine comprenait alors "deux maisons rurales,... une maison civile très dégradée composée de deux étages,... un petit jardin attenant complanté de quelques orangers,... trois citernes (dont) une au nord de la maison,... un moulin à huile pour le triturage des olives... $»^{14}$.

23 Aux Archives communales de Nice, l'espoir était de renseigner les constructions des années 20. Le répertoire des permis de construire conservés au dépôt de Cimiez évoquait bien des autorisations concernant Roseland à cette époque ${ }^{15}$. Espoir déçu puisqu'il s'agissait d'un projet de deux bâtiments de fermes situées ailleurs sur le domaine. Quant aux fonds des érudits niçois, ils n'apportaient pas d'éléments vraiment nouveaux ${ }^{16}$.

\section{Sources iconographiques}

Figure 5

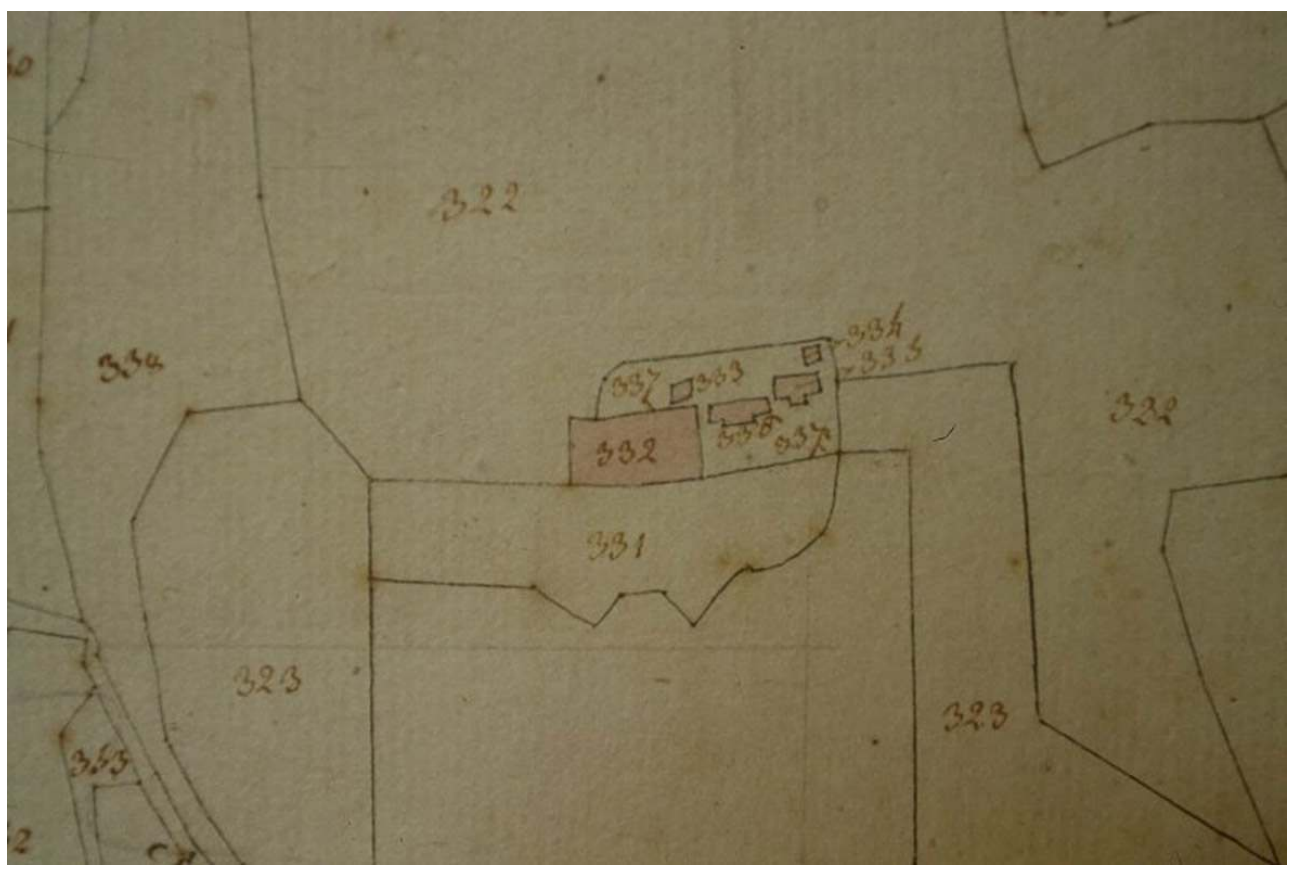

Plan cadastral ancien, section FF, 1812, Archives communales de Nice

Phot. J. Marx @ C.R.M.H. PACA., octobre 1993 
Totalement absentes à l'époque de l'Ancien Régime, elles se limitent avant le 20e siècle aux plans cadastraux anciens. Cette pauvreté est fréquente. En contrepartie Nice a la chance de disposer de deux plans cadastraux du 19e siècle, l'un levé pendant la période révolutionnaire au moment du premier rattachement à la France, le second après 1860, date du rattachement définitif. Sur le premier plan, daté 1812, la demeure figure sur la section FF dite de Barri Masson; sur le second, daté 1872, elle est en section P dite de Sainte-Hélène.

Figure 6

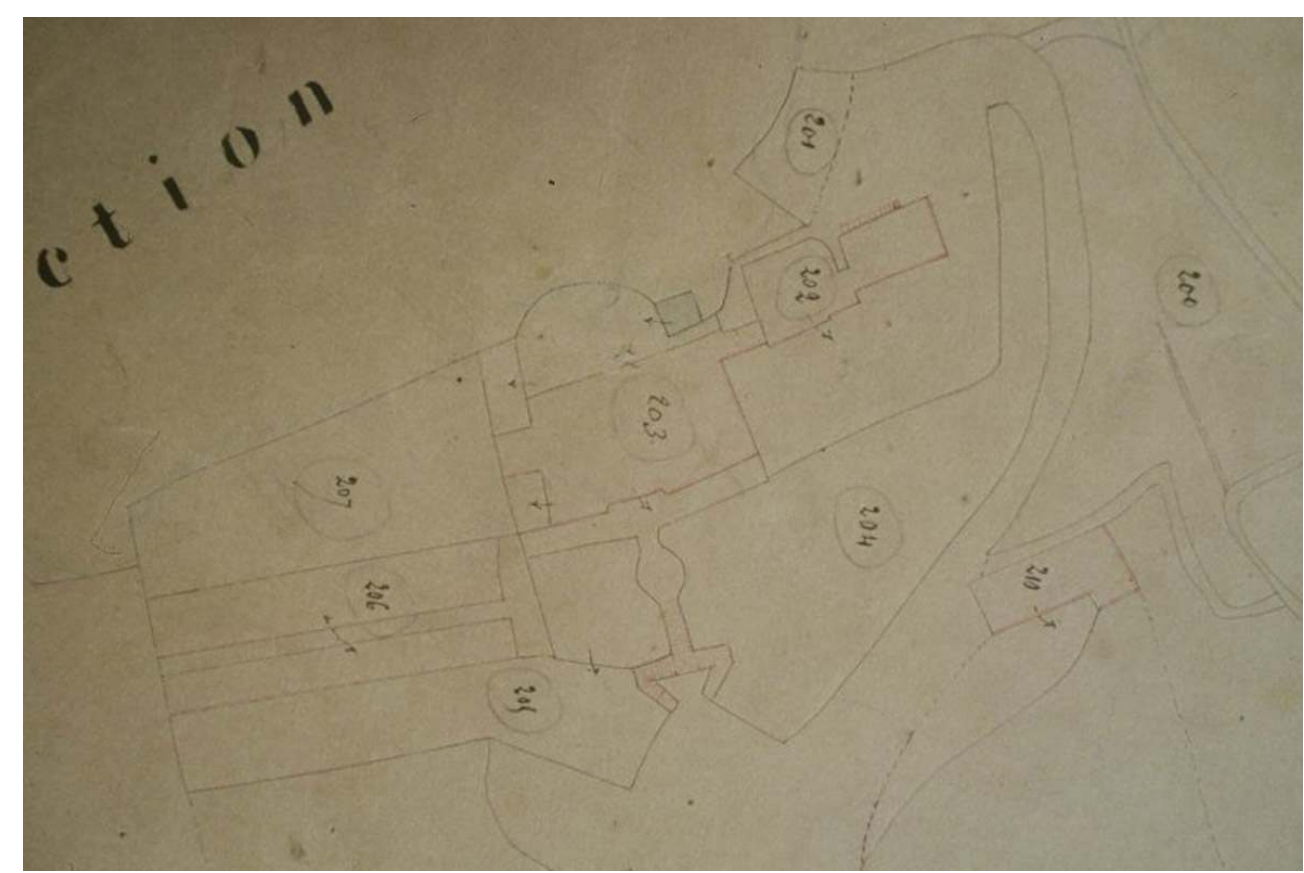

Second plan cadastral ancien, section P, 2e feuille, 1872, Archives communales de Nice

Phot. J. Marx (c) C.R.M.H. PACA., novembre 1993

La comparaison entre les deux plans et avec les dispositions actuelles est éloquente. Elle fait apparaître la permanence de l'organisation générale de la propriété et de ses jardins (abstraction faite bien sûr de la partie haute de Larcade), déjà en place au début du 19e siècle : axe général de l'accès sud-nord, tracé incurvé renvoyant à la cour circulaire au nord de la maison... Des différences sont néanmoins sensibles, par exemple dans l'organisation des dépendances, séparées puis reliées, ou dans la structure en terrasses des jardins; elles peuvent être imputables pour partie à des remaniements du 19e siècle, pour partie au traitement plus ou moins précis des deux plans.

En matière de documents graphiques, nous ne disposons d'aucun plan de l'édifice antérieur aux dernières décennies du 20e siècle. La Revue du Comminges a publié en 1978 un plan du cloître de Larcade ${ }^{17}$. Pour la demeure, l'étude du cabinet Février déjà évoquée contient onze relevés réalisés par le géomètre Matteudi en 1988, plans, coupes élévations ... dont les originaux semblent perdus. 


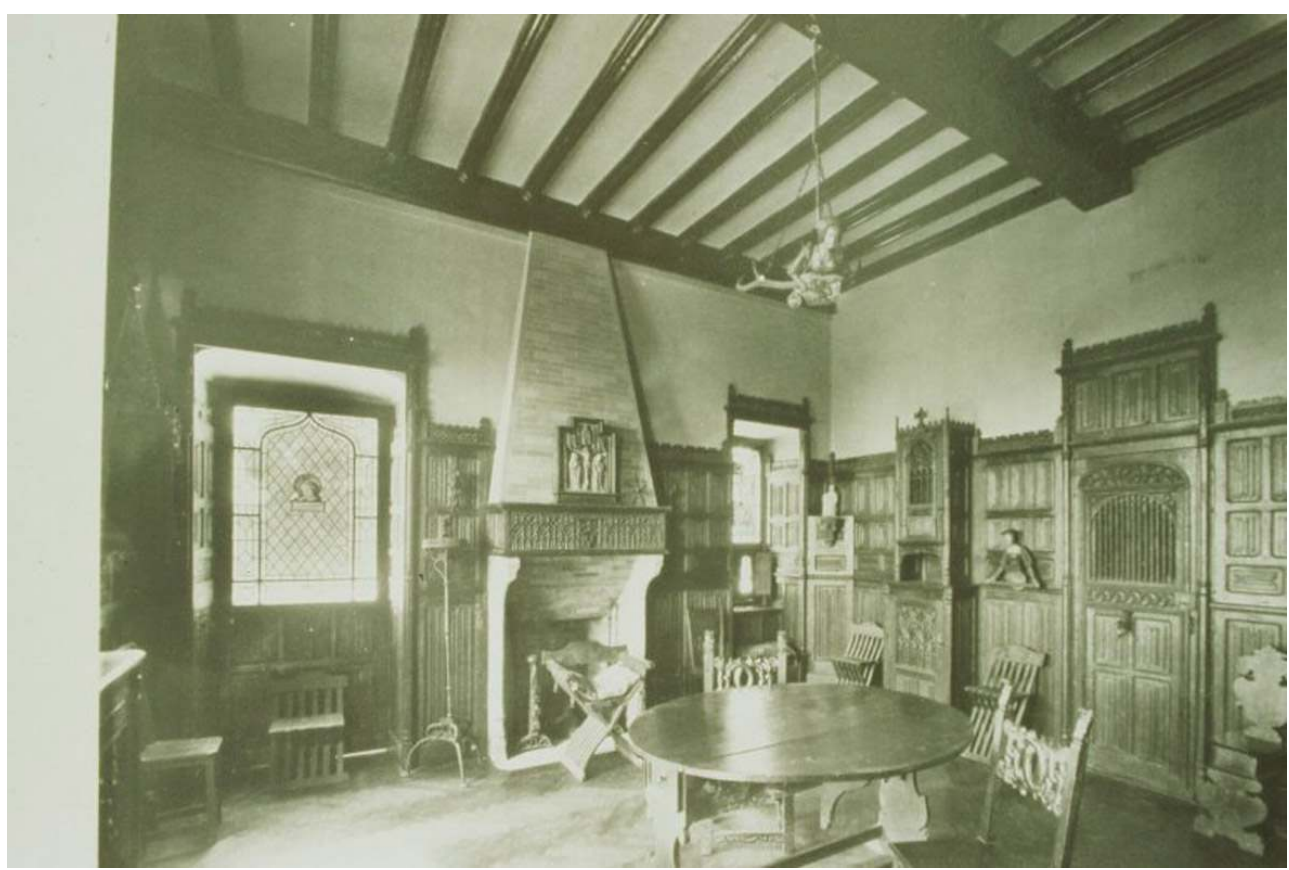

Photographie ancienne montrant la salle à manger au 1er étage, Paris, Ministère de la Culture, Archives photographiques, fonds Gilletta / GLT 0714 F

Repro. J. Marx @ C.R.M.H. PACA., mars 1994

L'iconographie du début du 20e siècle sur Roseland est assez riche. Elle permet de connaître l'état d'à peu près toutes les parties du domaine vers 1925-1930. Outre les photographies publiées dans les ouvrages et articles cités ${ }^{18}$, on possède un certain nombre de cartes postales anciennes ${ }^{19}$. Certaines ont été éditées à partir de clichés réalisés par une agence de photographie célèbre à Nice, les Gilletta. Par chance, les archives photographiques du ministère ont pu acquérir un fonds provenant des Gilletta, dans lequel on trouve une série de vues montrant l'abbaye de Roseland, la demeure et ses décors intérieurs, les jardins, les constructions de Larcade sur la partie haute... ${ }^{20}$

\section{Examen sur le terrain}

Evidemment les sources ne peuvent dispenser de l'examen direct et attentif de l'édifice, qui les complète, les corrobore ou parfois les infirme, qui renseigne aussi des parties non documentées. Dans le cas de Roseland, l'analyse sur le terrain a permis par exemple de déceler sous les décors et les reprises de la demeure par Larcade, ce qui appartenait à l'ancienne maison de campagne aristocratique: voûte en arc-de-cloître au rez-dechaussée sous une fausse croisée d'ogives, baies à larmier en chapeau de gendarme, baies et menuiseries chantournées semblables à celles rencontrées dans les maisons du 18e du vieux Nice, etc. Elle a montré aussi pour l'épiderme des façades la superposition des trompe-l'oeil peints sur l'enduit, trompe-l'oeil qui paraissent presque neufs sur les clichés des Gilletta. 


\section{Conclusions de l'étude et problèmes résiduels}

\section{Conclusions historiques}

Une des parties essentielles du dossier de recensement des monuments historiques s'intitule "Conclusions historiques". Cette partie correspond en fait à la globalité de l'historique de l'édifice, puisque à la différence des dossiers de l'Inventaire, cette partie intègre non seulement les données fournies par des sources indiscutables, mais aussi des interprétations, comparaisons ou hypothèses, permettant de dresser une histoire de l'édifice aussi linéaire que possible. Cette conception répond à la nécessité de faciliter le travail des utilisateurs du dossier. La contrepartie obligatoire en est la prudence qui doit présider aux affirmations et la précision des références. Dans le cas de Roseland, l'étude a conduit à des certitudes, permettant de compléter et de modifier l'état initial des connaissances.

Figure 8

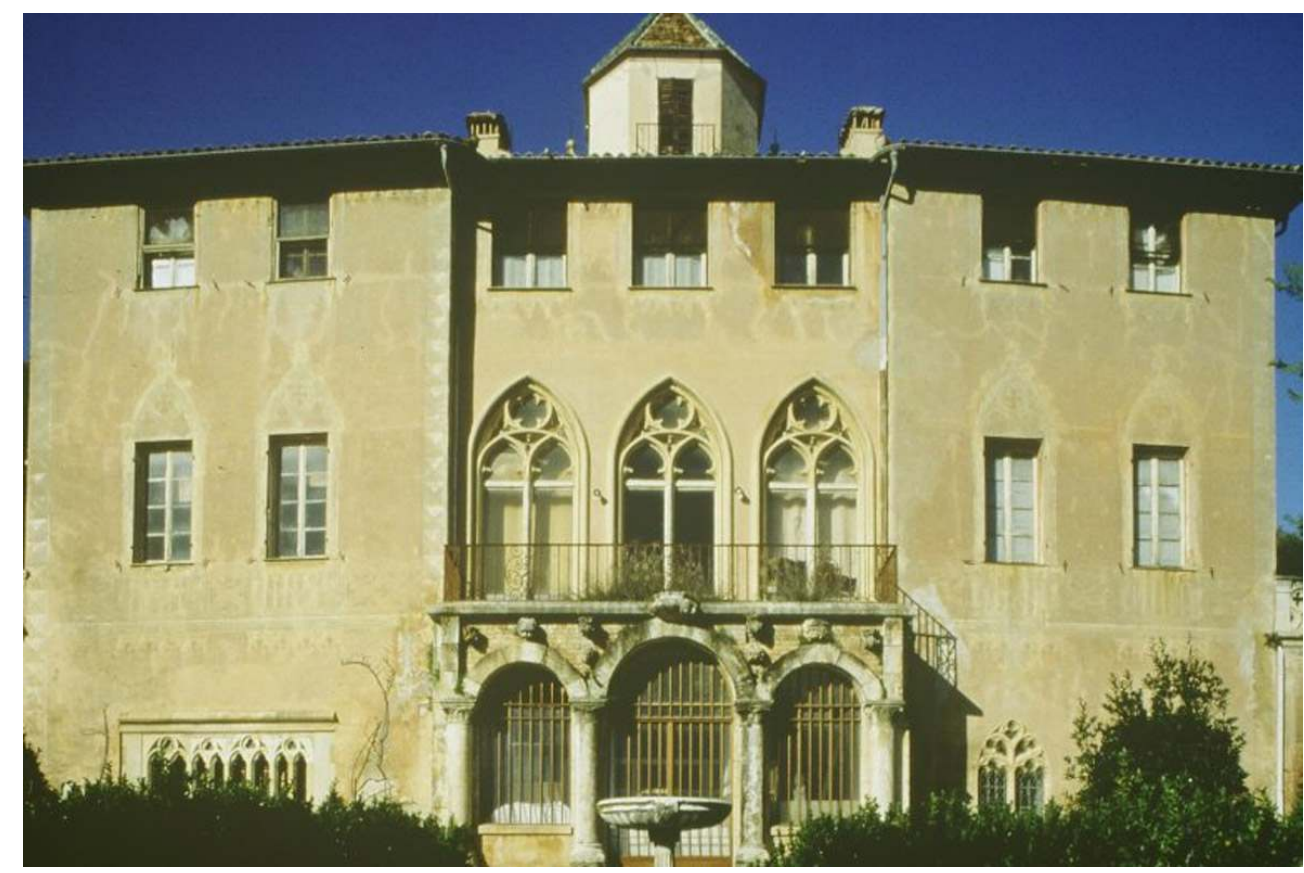

Façade principale sud de la demeure

Phot. J. Marx (@) C.R.M.H. PACA., novembre 1993

L'évolution du domaine ne peut être ici que résumée brièvement : il s'agit d'une demeure de campagne, propriété rurale d'une famille aristocratique niçoise (et non une abbaye, ni la demeure des évêques de Vintimille), les Dalmassi, attestée à partir du milieu 18e (testament de 1763), mais remontant sans doute au moins au 17e siècle si on en juge à la structure et au plan de la demeure. Cette propriété associait à une fonction résidentielle une vocation économique (huile et vin). 
Figure 9

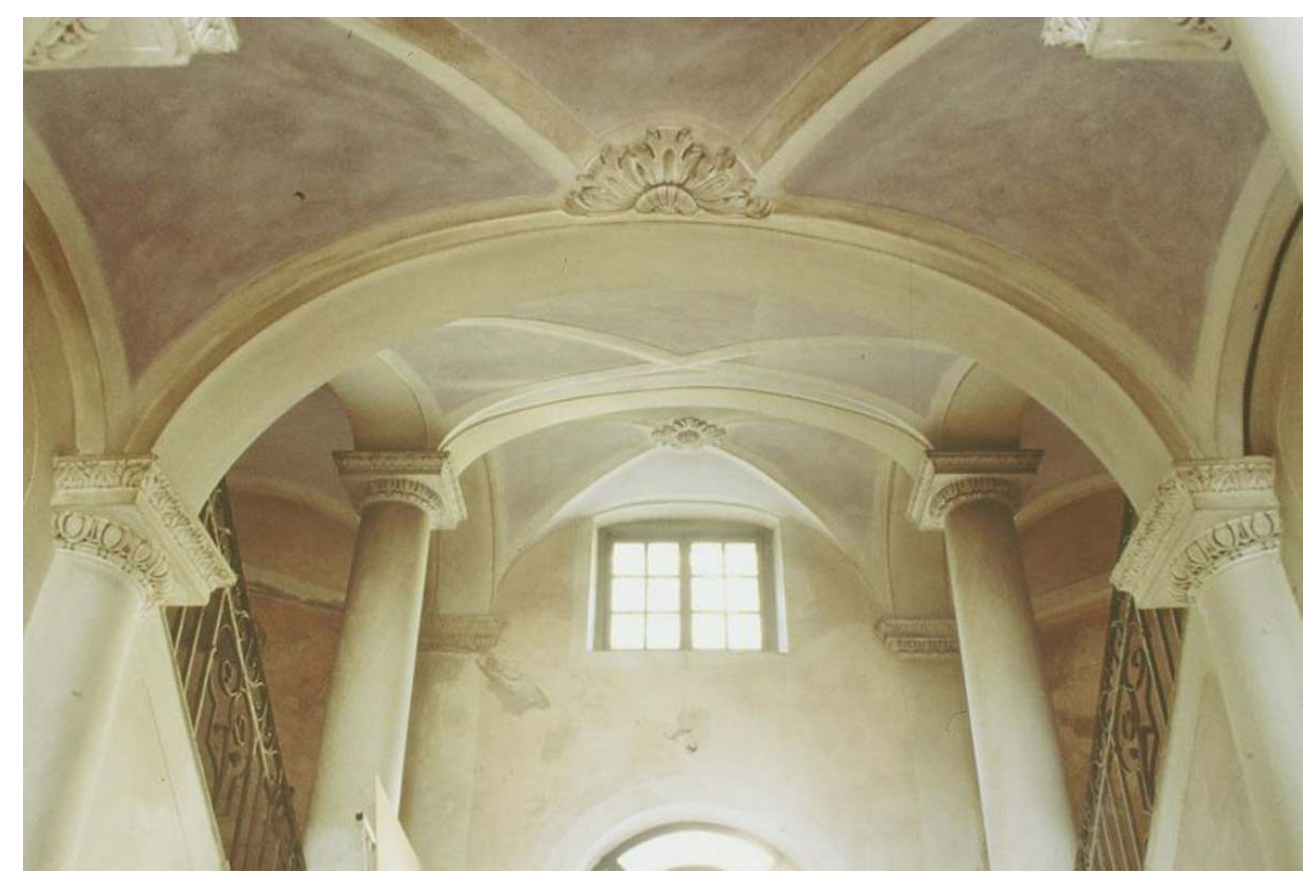

Escalier de la demeure, vu vers le nord

Phot. J. Marx (c) C.R.M.H. PACA., octobre 1993

31 Des modifications somme toute légères furent apportées au fil du 19e siècle, sur les décors par exemple, ou de manière peut-être plus importante sur les jardins (modification du parcellaire, création de l'accès oriental, étagement des terrasses). En revanche la transformation de l'édifice par l'antiquaire Edouard Larcade dans les années 20 fut vraiment générale. L'opération toucha la demeure (façades, intérieurs, décors), les dépendances, les jardins, et surtout la partie supérieure du domaine avec la création du pseudo-cloître, de la chapelle, d'une fausse ruine, permettant de présenter les riches collections lapidaires médiévales acquises par ce collectionneur. Ces travaux de Larcade se placent chronologiquement entre 1923 et $1927^{21}$, plus probablement après $1925^{22}$. 
Figure 10

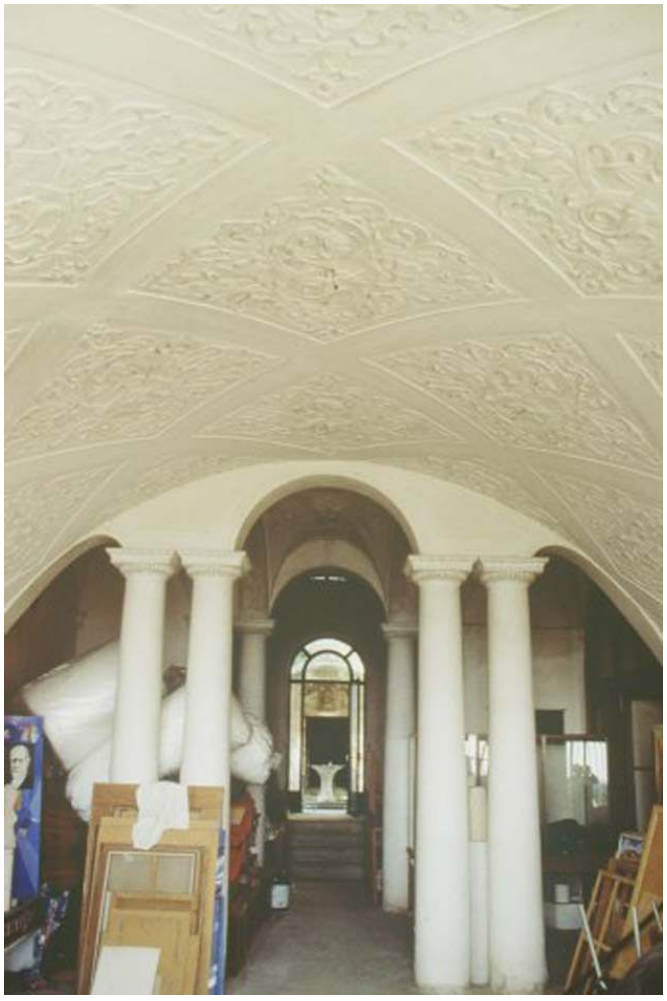

Vestibule de la demeure, vu vers la cour circulaire Phot. J. Marx @ C.R.M.H. PACA., octobre 1993 
Figure 11

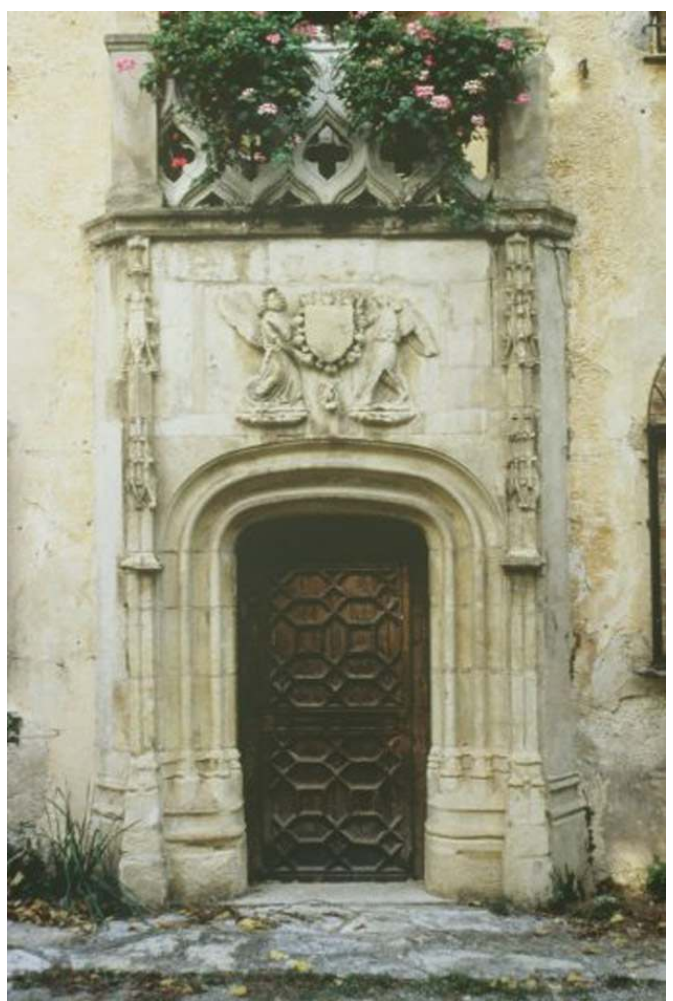

Bâtiment des dépendances, porte flamboyante remployée

Phot. J. Marx @ C.R.M.H. PACA., novembre 1993

Ils ont finalement conduit à un véritable détournement de sens de l'édifice, initialement représentatif d'un mode d'occupation de la campagne par l'aristocratie locale à l'époque moderne, maintenant lieu de collection de sculpture médiévale, évocateur du goût antiquaire des années 20 .

Si les grandes lignes sont connues, les incertitudes demeurent bien sûr nombreuses. Elles touchent aussi bien l'histoire de la propriété avant le 18e siècle, l'évolution architecturale de la demeure, la paternité du décor stuqué du rez-de-chaussée (remaniement ou interprétation), la provenance des décors remployés dans la maison, les dépendances et les jardins ${ }^{23}$, etc. A titre d'exemple, un point peut être tenté sur deux de ces zones d'ombre.

\section{Le problème de la cour arrière et de la grotte}




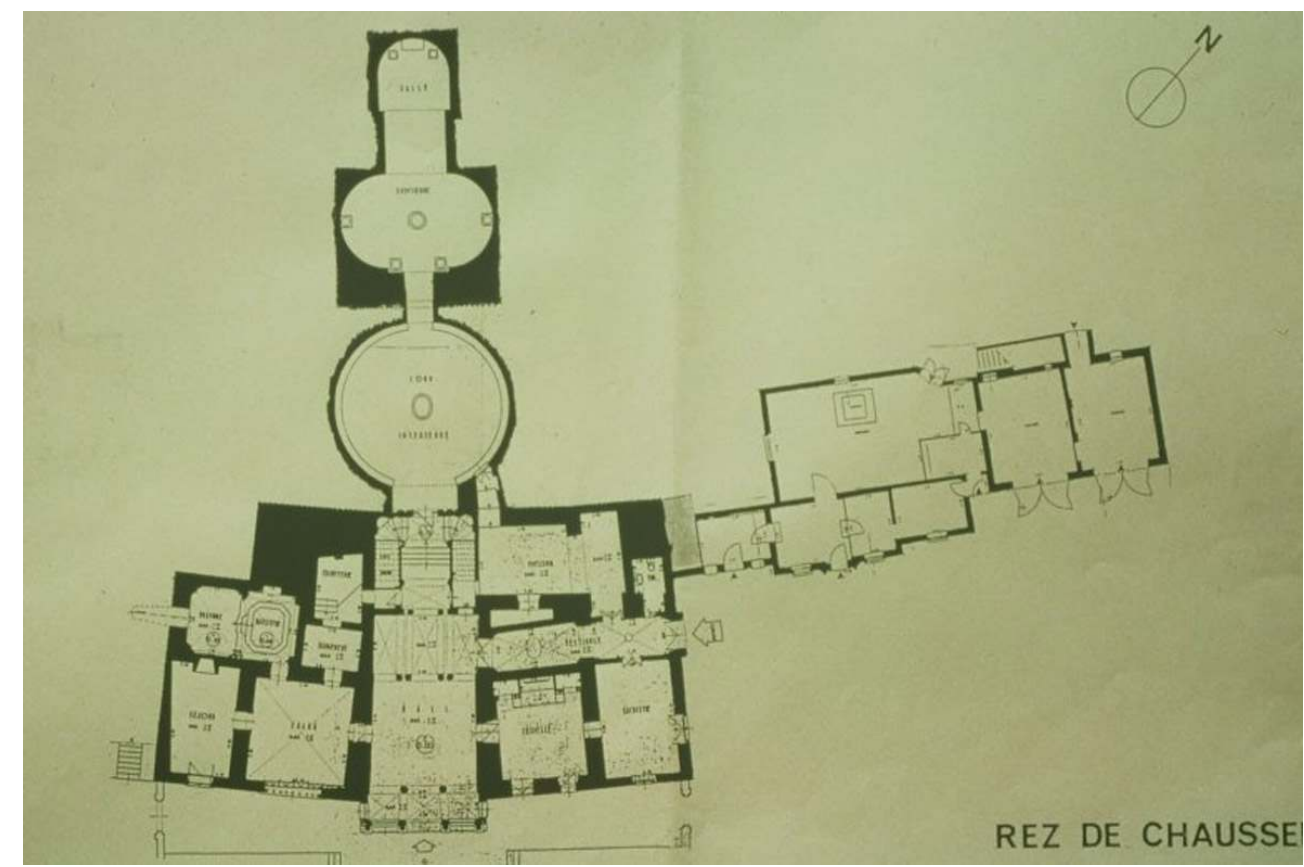

Plan du rez-de-chaussée de la demeure, montrant le dispositif cour-grotte à l'arrière, relevé Matteudi, ville de Nice, 1988

Phot. J. Marx ( C C.R.M.H. PACA., mars 1994

Un des axes principaux qui structurent le domaine de Roseland monte, nous l'avons dit, du sud au nord en passant par la demeure. A l'arrière de celle-ci, par l'escalier, il débouche sur un intéressant dispositif constitué d'une cour circulaire enterrée puis d'une grotte. La cour, totalement bordée d'un mur haut de quatre mètres, est dominée par une promenade circulaire au niveau de l'étage de la maison. La grotte est creusée dans la falaise de la terrasse supérieure. Elle offre un plan curieux : à une première partie élargie par des absides latérales et couverte d'une coupole ovale avec lunette au-dessus de la tribune, fait suite une sorte de large couloir voûté en anse-de-panier, puis une dernière partie un peu plus large terminée par une abside voûtée en cul-de-four. La façade est percée de deux portes superposées, l'une au niveau de la cour, l'autre ouvrant sur la promenade circulaire de l'étage, et elle est surmontée par un petit campanile. 
Figure 13

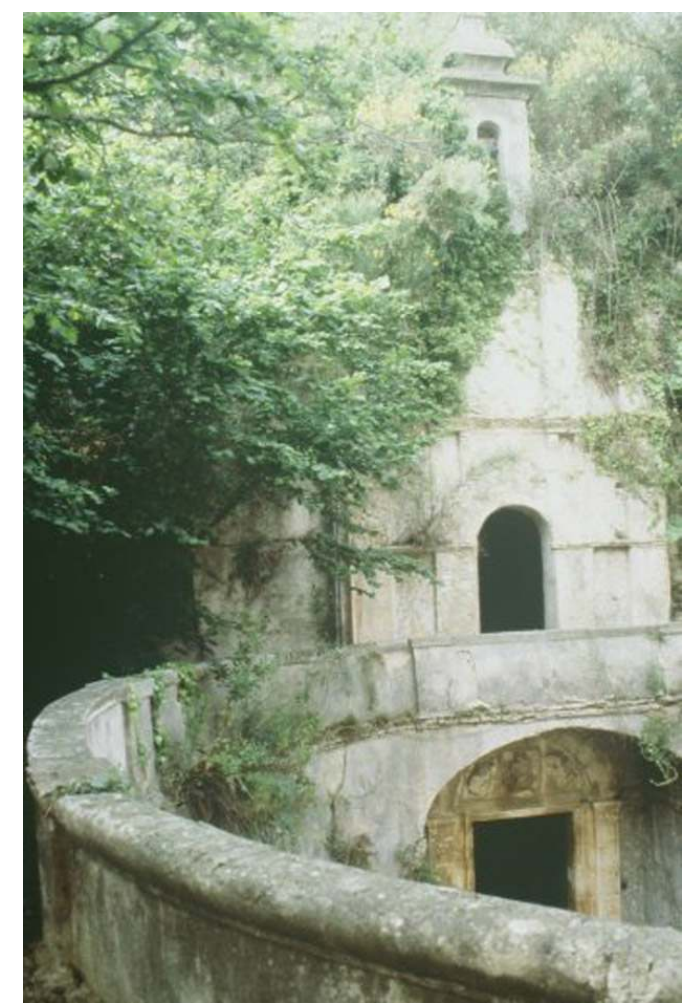

Vue partielle de la cour circulaire et de la façade de la grotte

Phot. J. Marx (C) C.R.M.H. PACA., mai 1992

L'interprétation comme la datation de cet ensemble posent problème. Les articles ou études qui l'évoquent parlent à son sujet de "citerne transformée en caveau égyptien " ( L'Espoir, 1957), ou de chapelle, de salle égyptienne (étude de la ville), de nymphée (mais il n'y a pas d'eau)... La présence du campanile, d'une sorte de tribune au-dessus de l'entrée et le plan en abside de l'extrémité septentrionale pourraient effectivement renvoyer à un usage cultuel, et une chapelle Saint-Pierre est bien attestée sur le site en 1757, sans que l'on puisse la localiser. Mais la grotte ne recèle apparemment ${ }^{24} \mathrm{ni}$ autel ni autre dispositif cultuel.

Le plan original de la grotte, unique à notre connaissance sur la Côte d'Azur, offre par ailleurs des parentés avec d'autres souterrains en France, telle la Bove des Chevaliers à Neuville-sur-Touques (Orne). Cette dernière avait une fonction ésotérique alors qu'ici rien de tel n'est connu. Bien que la piste d'un sens symbolique ne doive peut-être pas être écartée trop rapidement, il s'agit donc vraisemblablement d'une analogie formelle purement fortuite.

37 A Roseland, ni la cour ni la grotte ne sont documentées par les sources, mais le tracé curviligne des plans cadastraux anciens atteste certainement leur présence au moins depuis le début du 19e siècle. Il ne s'agit donc pas d'une création de Larcade. En fait l'analyse du parti architectural du binôme cour enterrée/grotte comme celui des composantes de la façade de la grotte renvoient à une interprétation d'architecture de jardin. Il s'agit sans doute à l'origine d'une sorte de grotte de fraîcheur, probablement imputable à l'époque maniériste. Les modèles en sont incontestablement italiens: on pense au nymphée de la villa Giulia ou à d'autres exemples romains. Des incertitudes 
demeurent cependant sur les dispositions d'origine, et il est d'ailleurs probable que celles-ci ont changé avec l'évolution de l'usage. Une étude plus approfondie devrait permettre de les lever en partie lorsque les conditions de visite le permettront.

\section{Le cloître de Larcade}

Figure 14

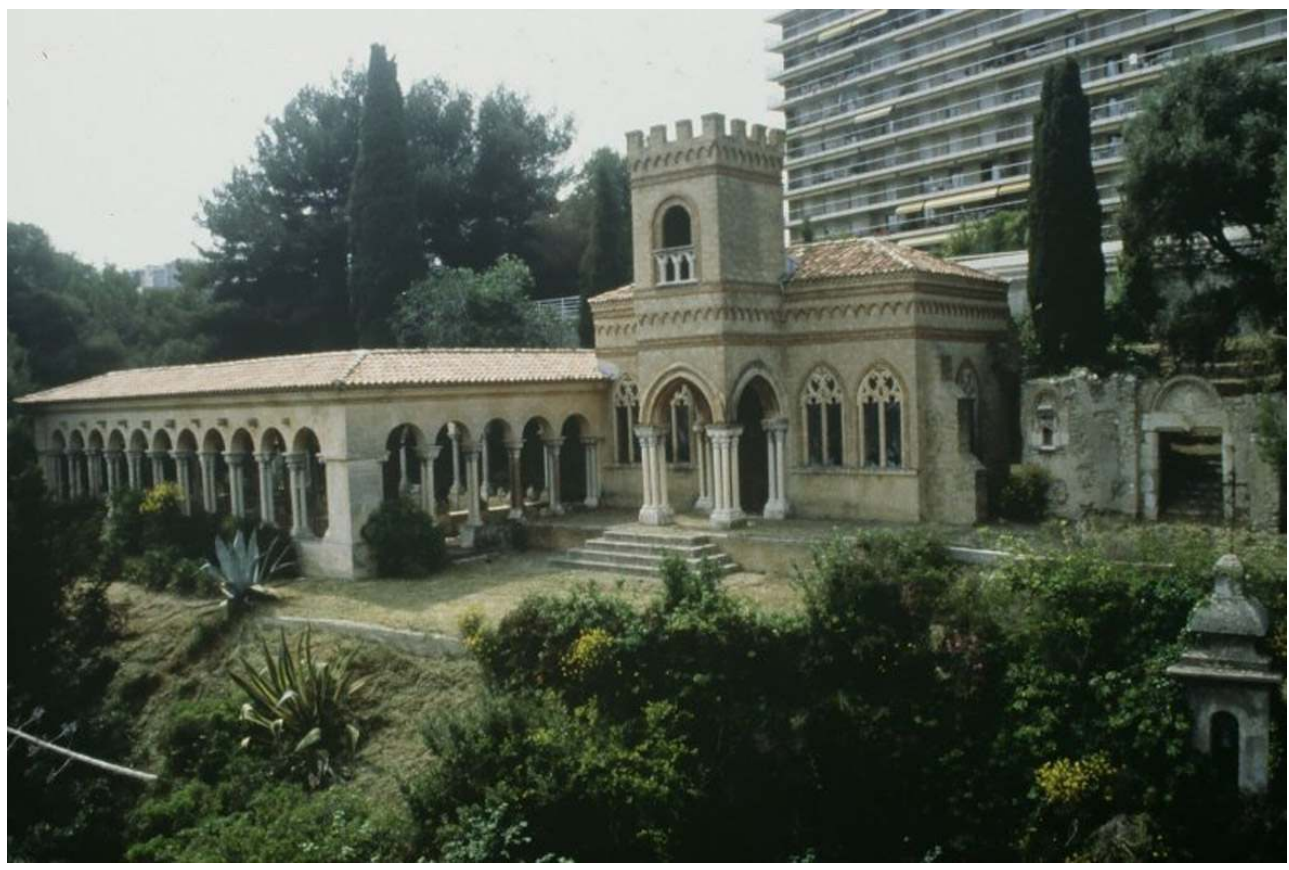

Ensemble des constructions de Larcade sur la terrasse haute

Phot. J. Marx (C C.R.M.H. PACA., mai 1992

Les constructions de la terrasse supérieure comportent un cloître factice ouvert sur l'extérieur et sur la mer, une chapelle et une fausse ruine. Elles ont été bâties en 1925-1926 par l'antiquaire Edouard Larcade ${ }^{25}$. Il est possible que le paysagiste Octave Godard, un élève d'Edouard André, ait œuvré non seulement aux jardins mais aussi à la construction du cloître car le travail sur les sols des galeries est caractéristique de son style.

Cet ensemble n'avait aucun rôle fonctionnel. Il s'agit d'une architecture prétexte pour l'exposition de nombreux éléments de la riche collection lapidaire de l'antiquaire, complément des nombreux remplois rencontrés ailleurs dans la demeure, les dépendances et les jardins. A la différence de ces derniers, dont l'inventaire et l'analyse restent à faire, les éléments intégrés dans l'ensemble de la terrasse supérieure ont été beaucoup étudiés, mais les conclusions des différents auteurs ne concordent pas toujours parfaitement. Le dossier de recensement a permis d'établir un état de la question relativement clair. 
Figure 15

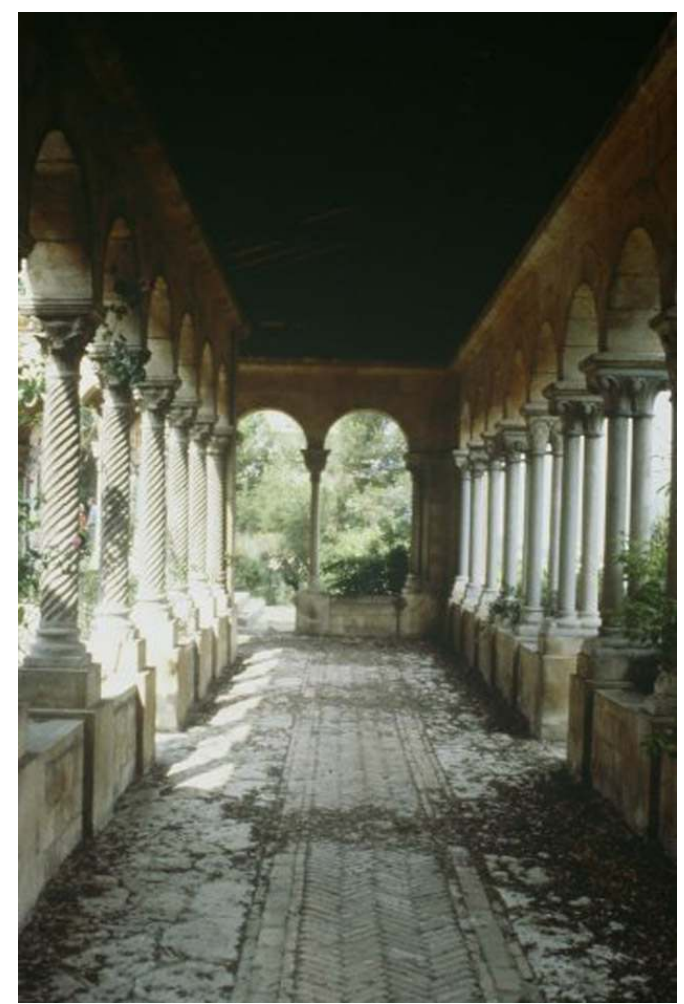

Galerie sud du cloître factice

Phot. J. Marx (c) C.R.M.H. PACA., mai 1992

Ouvert sur trois côtés, le cloître est constitué de deux séries de colonnes à chapiteaux. Toutes sont des remplois anciens, mais elles ne sont pas homogènes. Les deux séries appartiennent à des époques et à des édifices différents.

Pour la colonnade extérieure, comme pour la chapelle voisine, les monuments "donateurs" sont des édifices médiévaux du Sud-Ouest de la France. Traditionnellement ces chapiteaux étaient attribués au cloître de Bonnefont. Dans un article publié en $1980{ }^{26}$, Elie de Comminges a fait la démonstration qu'ils ne pouvaient en provenir, du moins pas tous. Il a repéré treize types distincts et jugé qu'ils appartenaient à d'autres édifices du Sud-Ouest, certains non identifiés. Parmi les attributions proposées, on relève l'église de L'Isle-Jourdain, l'abbaye de Lescaladieu, le cloître de Lavedan, etc. Les problèmes de provenance sont loin d'être tous réglés et le recours à un spécialiste de la sculpture médiévale du Sud-Ouest comme Jean Cabanot s'avérerait certainement fructueux. 
Figure 16

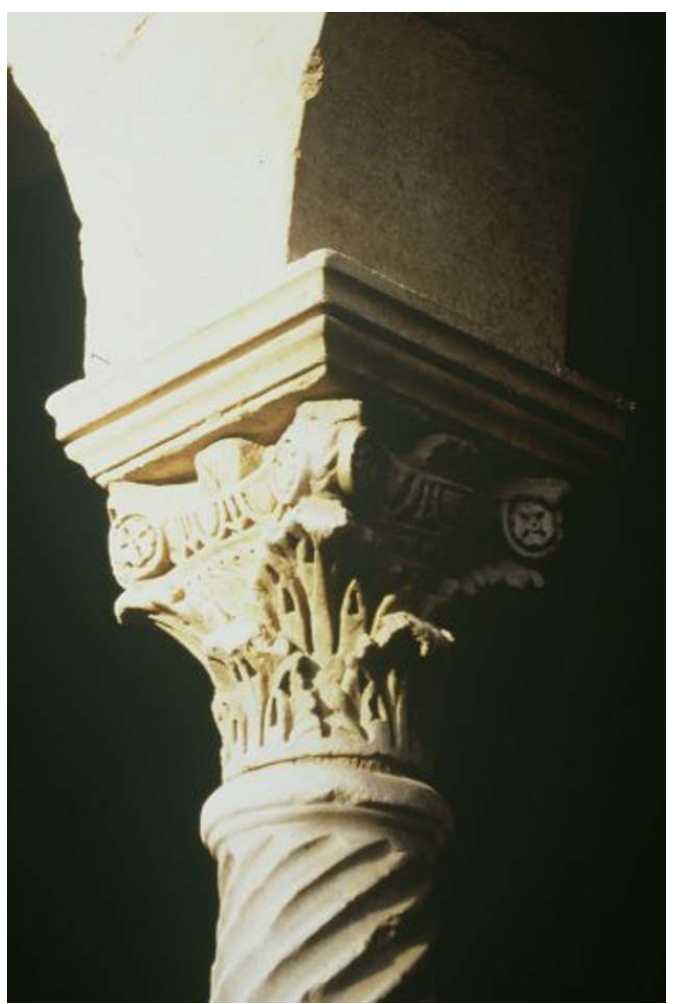

Un des chapiteaux de la colonnade intérieure du cloître, provenant de l'ancienne église de la Daurade de Toulouse

Phot. J. Marx @ C.R.M.H. PACA., octobre 1993 chapiteaux proviennent d'un édifice insigne de l'histoire de l'art du Midi toulousain, la vieille église de la Daurade de Toulouse, et qu'ils remontent à l'époque paléochrétienne, probablement au 4e ou au 5e siècle ${ }^{27}$.

Passons sur les origines controversées de la primitive église Sainte-Marie de la Daurade, temple antique remployé ou édifice cultuel paléochrétien. Son chœur avait la forme d'un polygone irrégulier à trois rangs superposés de niches ornées de mosaïques, séparées par des colonnettes de marbre blanc, à cannelures torses ou à pampres, portant des chapiteaux corinthiens, composites ou ioniques ${ }^{28}$. L'église fut démolie en 1761 , mais les colonnes furent en partie amenées par J-F. de Montégut, conseiller au parlement de Toulouse, dans le parc de son château de Montégut-Ségla, près de Muret. Il les remploya dans une sorte de temple dédié à Apollon, inauguré en 1787, qui subsista jusqu'en $187{ }^{29}$. Une partie des colonnes disparut alors, d'autres furent transférées dans le vestibule du château jusqu'à sa vente en 1912. On en retrouve aujourd'hui deux au Louvre, d'autres en Amérique, quelques-unes sont revenues au musée des Augustins de Toulouse, mais l'ensemble le plus conséquent fut racheté par Larcade. Ces colonnes en marbre blanc avec leurs chapiteaux constituent la colonnade intérieure du cloître de Roseland.

Se pose en conclusion la question de la spécificité de la monographie d'édifice telle qu'elle est pratiquée dans les dossiers de recensement des monuments historiques. Cette spécificité existe-t-elle? Ne réside-t-elle pas principalement dans le fait que, justement, lesdits dossiers sont nécessairement des monographies, dans un contexte de la recherche 
qui conduit au contraire bien souvent aujourd'hui à privilégier l'approche sérielle, jugée plus apte à renouveler les connaissances en histoire de l'art ?

L'une de ses caractéristiques tient en tout cas aux limites de l'étude, répondant à des objectifs déterminés et disposant d'un délai imparti relativement rigoureux. La conséquence inéluctable en est que ce type de monographies, produit d'une vision rapide et globalisante, n'est pas totalement abouti. Elles posent souvent plus de questions qu'elles n'en résolvent, et Roseland participe de cette insatisfaction. Ce regret touche aussi à l'impossibilité d'élargir l'étude. A la différence d'études de type universitaire, le temps manque pour comparer, pour inscrire le monument dans un corpus. Le défaut principal de nos monographies est sans doute d'être trop des monographies. Cette difficulté réductrice devrait toutefois être progressivement aplanie grâce au développement des outils modernes de connaissance et de diffusion du patrimoine, permettant de situer plus rapidement un édifice dans sa famille.

Corollaire de la démarche, un autre élément fort est le lien à la réalité vivante. La protection -donc le dossier qui l'étaie- résulte en effet toujours du croisement de l'intérêt architectural, artistique ou historique avec des paramètres d'opportunité, de faisabilité qui sont inhérents à l'insertion du monument dans la société. Le dossier fait place à l'écoute des partenaires, il porte attention au vécu du monument, établit un trait d'union entre l'édifice et l'homme. A la croisée d'approches multiples, nécessairement pluridisciplinaires, il ne peut se satisfaire du seul regard de l'historien d'art et de l'historien. Même si la restitution de cette dimension humaine est difficile, sa prise en considération est vraiment essentielle.

On sait maintenant que le patrimoine est choix de société. Qu'il ne peut être jugé seulement comme valeur intrinsèque, absolue, mais comme reflet des nostalgies, des pulsions, des combats d'une époque. Reflet de notre propre subjectivité donc, et l'on abuse sans doute à propos de son étude de l'adjectif "scientifique". Le patrimoine est choix, et ce choix, reflétant l'évolution du goût, révèle une certaine labilité des critères. Roseland aurait été refusée il y a peu et son classement a été discuté ardemment tant en commission régionale qu'en commission supérieure: allait-on cautionner le pillage, valoriser l'elginisme ?30

Une frustration imputable à un cadre temporel et spatial contraignant, compensée par une large ouverture sur la dimension humaine du patrimoine, tels sont sans doute deux des caractères qui contribuent à la spécificité d'un dossier monographique des monuments historiques.

\section{NOTES}

1. Dossier du 23 février 1994, concluant un travail commencé en 1993 (archives de la Conservation régionale des Monuments historiques)

2. Depuis le 30 septembre 1971, "l'abbaye de Roseland" appartient à la commune de Nice et est affectée au service culturel de la ville 
3. Le dossier a été présenté à la CO.RE.P.H.A.E. de PACA. le 17 mars 1994 et à la Commission supérieure des Monuments historiques le 15 janvier 1996

4. Notamment Recensement des immeubles pour une protection au titre des monuments historiques, manuel méthodologique, Direction du Patrimoine, janvier 1996

5. L'Illustration, $\mathrm{n}^{\circ} 4481,19$ janvier 1929

6. L'Espoir, 24 août 1957

7. Reflets des Alpes Maritimes, $n^{\circ} 4,1980$

8. Godard (Octave).- Les jardins de la Côte d'Azur. Paris, imprimerie J. Dumoulin, 1927 p. 6 et pl.I, II, III

9. J.B/-L'ancienne abbaye de Roseland à Nice. L'Illustration, $\mathrm{n}^{\circ} 4481,87 \mathrm{e}$ année, 19 janvier 1929 , p. 62 et 8 ill

10. Boursier-Mougenot (E.J.P.) - Recensement des parcs et jardins remarquables des AlpesMaritimes. Conseil Général des Alpes Maritimes, 1992

11. Allegre (Victor).- Des colonnes commingeoises à l'abbaye de Roseland à Nice. Revue du Comminges, 1974, volume LXXXVII, p. 373-378

Comminges (Elie de).- Le cloître de Roseland. Revue du Comminges, 2ème trimestre 1980, tome XCIII, p. 213-225

12. Rey (Raymond).- Le sanctuaire paléo-chrétien de la Daurade à Toulouse et ses origines orientales. Annales du Midi, LXI, 1949, p. 249-273

- Mesple (Paul).- Recherches sur l'ancienne église de la Daurade. Mémoires de la Société Archéologique du Midi de la France, XXXI, 1965, p. 41-56, 109 et pl. V-VII

- Labrousse (Michel).- Toulouse antique. B.E.F.A.R. 212. Paris, De Broccard, 1968, p. 412-417

- Durliat (Marcel), Deroo (Christophe), Scelles (Maurice).- Recueil général des monuments en France pendant le Haut Moyen Age (IVe-Xe siècles), tome IV : Haute Garonne. Paris, Editions du C.T.H.S., 1987, p. 12-13, 28, 89-99, 140-141 et pl. LVII à LXIV

- Musée Saint-Raymond de Toulouse.- Palladia Tolosa, Toulouse romaine. Catalogue d'exposition, novembre 1988-mars 1989, p. 141-146

13. A.D. Alpes-Maritimes, B 303.- Sénat de Nice: protocoles ou recueils des minutes des testaments... (registre, 1742-1765), fol.272-285, testament du 30 juillet 1763

14. A.D. Alpes-Maritimes, Q 75. - Procès-verbaux d'estimation et de vente, $n^{\circ}$ 401-450, brumaire an $\mathrm{V}$ : Vente $\mathrm{n}^{\circ} 435$ du 9 brumaire à un nommé Pierre Hulin

15. A.C. Nice (dépôt de Cimiez), 2 T 414).- Permis de construire, 1925

16. Par exemple Fonds Louis Cappati, 2.S./231.-Villas : N-Z. Pour Roseland il contient notamment des coupures de presse et quelques références

17. Revue du Comminges, 1978, p. 142

18. Ouvrage de Godard, article de l'Illustration, article de l'Eclaireur du Dimanche illustré, en 1932, etc.

19. Une dizaine est conservée dans les collections du musée Lascaris à Nice

20. Archives photographiques de la Direction du Patrimoine, fonds Jean Gilletta (1856-1933)

21. Respectivement dates de l'achat de la propriété par Larcade et du livre de Godard qui les montre

22. Ces parties ne figurent pas en effet sur le plan annexé au permis de construire une ferme. Cf. supra

23. Ce problème est très fréquent dans l'architecture de la villégiature sur la Côte d'Azur, où les moyens financiers des commanditaires leur ont permis d'accumuler des collections parfois considérables. Voir par exemple le cas analogue de la Villa Ephrussi de Rothschild au cap Ferrat

24. Sous réserve d'un examen plus approfondi que l'état d'encombrement du lieu au moment du dossier ne permettait pas

25. Notice sur Edouard Larcade. Les Donateurs du Louvre, catalogue d'exposition de la R.M.N., Paris, Louvre, 1983, p. 247 
26. Comminges (Elie de).- Le cloître de Roseland, Revue du Comminges, tome XLIII, 2e trimestre 1980

27. Selon les auteurs, la datation fluctue du $4 \mathrm{e}$ ou $6 \mathrm{e}$ siècle

28. Une gravure représentant ce chœur est publiée dans : Martin (Dom Jacques).- La religion des Gaulois, tirée des plus pures sources de l'Antiquité. Paris, 1727, t. I, ch. XVIII, p. 146-172 et pl. 4

29. Cf. bibliographie donnée en note 12. Une inscription lapidaire en langue latine conservée à Roseland apporte des précisions sur ces événements

30. L'abbaye de Roseland a été classée en totalité par arrêté ministériel du 3 septembre 1996

\section{INDEX}

Mots-clés : inventaire général, en ligne, journal, revue électronique, revue numérique, périodique, patrimoine, histoire de l'art, France, architecture, Provence-Alpes-Côte d'Azur Keywords : on line, electronic journal, ejournal, heritage, history of art, dwellings

\section{AUTEUR}

\section{JEAN MARX}

Chargé d'études documentaires principal, Conservation régionale des Monuments historiques, Direction régionale des Affaires culturelles 23, boulevard du Roy René 13100 Aix-en-Provence. jean.marx@culture.gouv.fr 